LA-UR- $94-271$

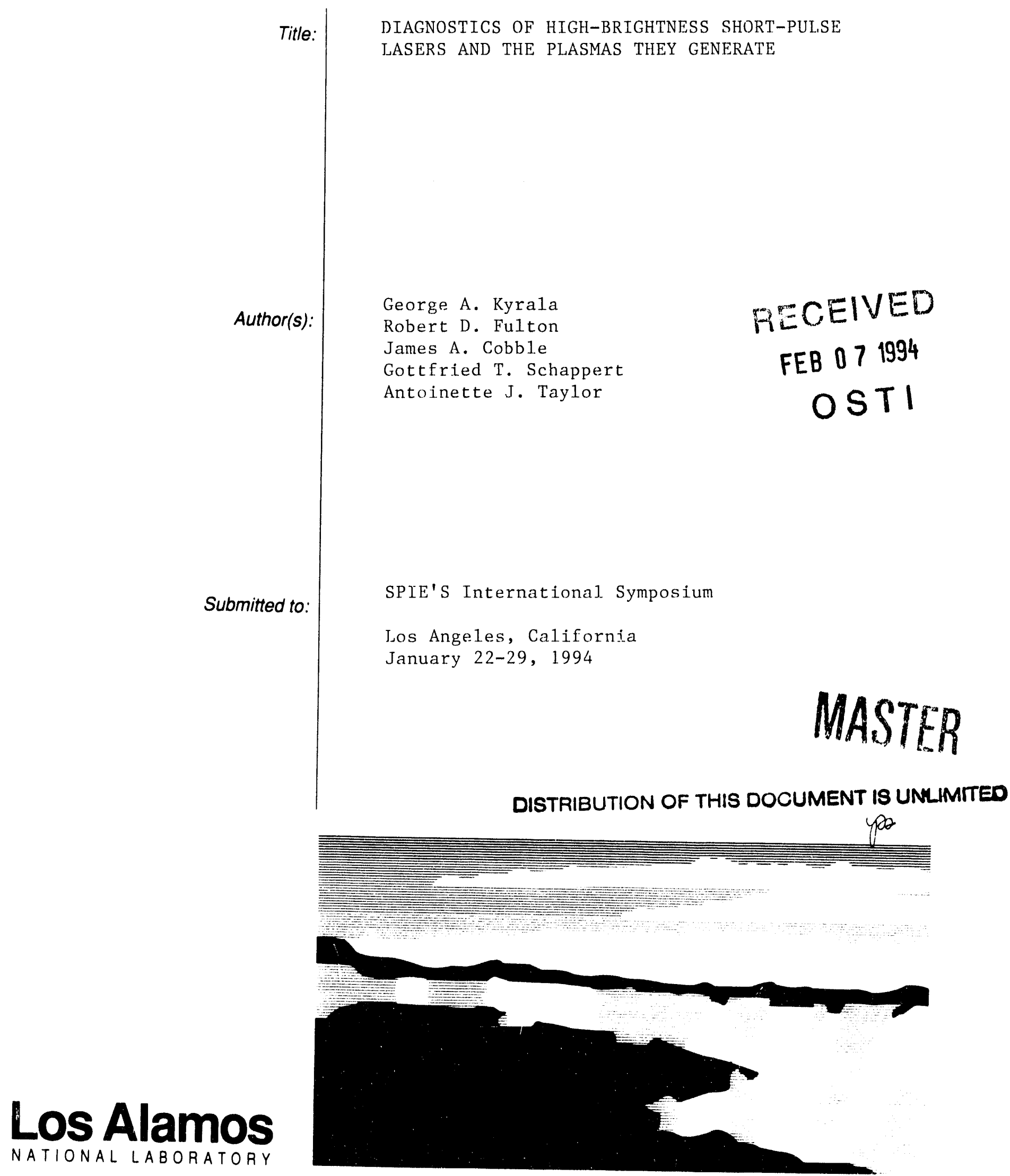

Los Alamos National Laboratory, an aftirmative action/equal opportunity employer, is operated by the University of California for the U.S. Department of Energy under contract W-7405-ENG-36. By acceptance of this article, the publisher recognizes that the U.S. Government retains a nonexclusive, royalty-free license to requests that the publisher identify this article contribution, or to allow oihers to do so, for U.S. Government purposes. The Los Alamos National Laboratory requests that the publisher identify this article as work performed under the auspices of the U.S. Departmerit of Energy. 


\title{
Diagnostics of high-brightness short-pulse lasers and the plasmas they generate
}

\section{George A. Kyrala, Robert D. Fulton, Jim A. Cobble, Gottfried T. Schappert aná Antoinette J. Taylor}

\author{
Los Alamos National Laboratory \\ Mail Stop E-526 \\ Los Alamos, NM 87545
}

\begin{abstract}
The properties of a laser influence the interaction of the intense laser light with materials. We will describe some of the diagnostics that we have implemented at the Los Alamos Bright Source to correlate the changes in the $\mathrm{x}$-ray spectrum and temporal history of a laser generated silicon plasmas with the changes of the incident $\mathrm{XeCl}$ laser light. One property is of special interest, the existence of a short prepulse. We find that the prepulse enhances the generation of the $x$-rays from a later pulse.
\end{abstract}

\section{INTRODUCTION}

Efficient production of high energy $x$-rays during the interaction of laser light with matter have interested us for many years. The variation and spectrum of these $x$-rays have been used to diagnose the state of the plasmas and hence the laser interactions. We are also interested in using these very intense $x$ rays for performing non-linear interactions with material and hence must characterize these $\mathrm{x}$-rays well. During the interaction of intense laser light with plasmas, the various properties of the laser light affect the interaction. Of these properties: energy, irradiance, prepulse, and pulse length have been systematically investigated. In the present study we will investigate the effect of a large short pulse-length prepulse on the generation of $x$-rays from a silicon plasma. The prepulses are generated some tens of picoseconds ahead of the main laser pulse, and irradiate the target at the same focus, thus allowing the solid surface to expand into a lower density plasma that interacts with the second pulse.

\section{DESCRIPTION OF THE LASER SYSTEM}

The laser system is a terawatt-class laser based on the amplification of subpicosecond pulses in $\mathrm{XeCl}$ discharge amplifiers. The laser system, ${ }^{1}$ shown in Figure 1, consists of a front-end which generates 308 -nm seed pulses by frequency-doubling the output of a synchronously-pumped subpicosecond dye oscillator-amplifier system, operating at $616 \mathrm{~nm}$. These UV seed pulses are amplified by an amplifier chain consisting of a small-aperture $\left(1-\mathrm{cm}^{2}\right) \mathrm{XeCl}$ preamplifier, then cleaned by a beam expanding vacuum spatial filter, folowed by amplification in a large aperture $\left(100-\mathrm{cm}^{2}\right)$ final amplifier. This system produces $250-\mathrm{mJ}, 315-\mathrm{fsec}$ pulses at a sustained repetition rate of $1 \mathrm{~Hz}$. Using $\mathrm{f} / 3.7 \mathrm{optics}$, we measure FWHM focal-spot dimensions of $3.4 \mu \mathrm{m} \times 4.1 \mu \mathrm{m}$ for the full-energy final output beam and therefore demonstrate a true focal-volume irradiance of $4.9 \times 10^{18} \mathrm{~W} / \mathrm{cm}^{2}$

\section{DISCLAIMER}

This report was prepared as an account of work sponsored by an agency of the United States Government. Neither the United States Government nor any agency thereof, nor any of their employees, makes any warranty, express or implied, or assumes any legal liability or responsibility for the accuracy, completeness, or usefulness of any information, apparatus, product, or process disclosed, or represents that its use would not infringe privately owned rights. Reference herein to any specific commercial product, process, or service by trade name, trademark, manufacturer, or otherwise does not necessarily constitute or imply its endorsement, recommendation, or favoring by the United States Government or any agency thereof. The views and opinions of authors expressed herein do not necessarily state or reflect those of the United States Government or any agency thereof. 
Fig. 1. Schematic of the laser system.

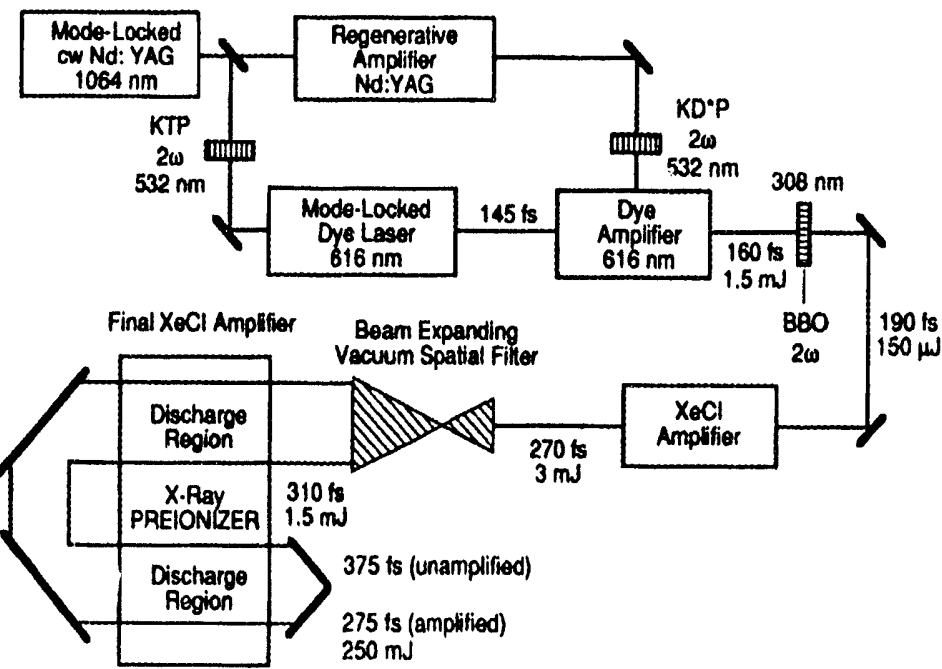

\section{DESCRIPTION OF THE EXPE RIMENTAL APPARATUS}

The experimental apparatus is shown in Figure 2. The Bright Source laser pulse is introduced into a UHV target chamber and focused by an $\mathrm{f} / 1.7$ off-axis parabola, with a focal length of $18 \mathrm{~cm}$, onto a solid flat target. The entrance window is an uncoated $1 \mathrm{~cm}$ thick UV grade fused silica to minimize the effects of self-focusing. The parabola is protected from target debris created in the laser-solid interaction by a $.25 \mu \mathrm{m}$ thick fused silica blast shield. Orientation of the parabola to yield best focus was determined off line and is reproduced in the target chamber by a retro-reflection off the rear surface to an accuracy of $\pm 17 \mu \mathrm{rad}$. This allows focusing of the original $10 \mathrm{~cm} \times 4 \mathrm{~cm}$ beam to a $5 \mu \mathrm{m}$ diameter focal spot containing $50 \%$ of the energy albeit with significant intensity variations within the focal spot. (include figure of focal spot measurement?) Once the angular alignment is set, the "best focus" is determined by translating the target along the central ray of the parabola until maximal $x$-ray yield, as determined by a set of filtered pin diodes, is achieved. The width of the $x$-ray yield, as a function of axial distance, is typically $100 \mu \mathrm{m}$. This width agrees with the Rayleigh range calculated for the focusing optic.

Outside the target chamber are a number of diagnostics used to characterize the incident laser pulse as to energy content, contrast ratio of the main pulse versus the ASE prelase, optical beam quality, time history and spectra of the incident and back scattered laser light. These quantities are essential in the determination of the irradiance on target and the target conditions created by the prelase fraction. Earlier work ${ }^{2,3}$ on solid targets and thin foils has demonstrated the critical influence of prelase on $x$-ray yield due to the importance of the scale length of the preformed plasma that the main pulse interacts with

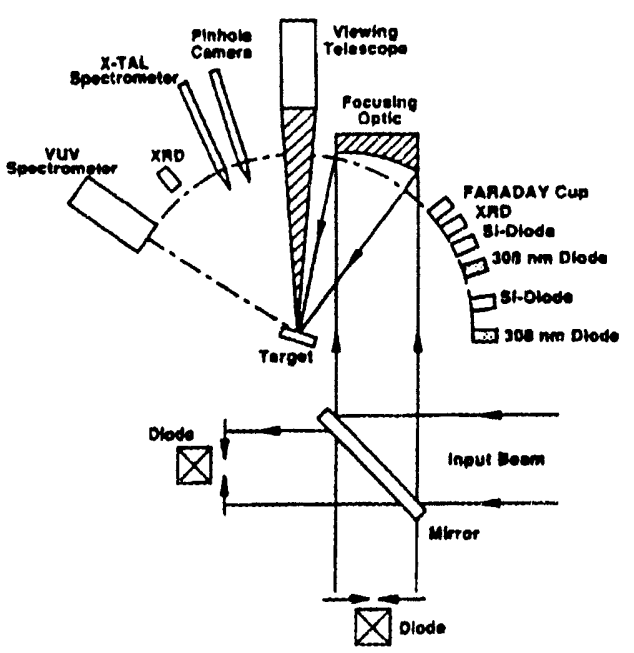

Fig. 2. Schematic of the experimental apparatus 
Wit.in the target chamber is the focusing optic, 3-axis target manipulator, and other, primarily $x$-ray, diagnostics. The target consists of a single crystal of silicon oriented in the 001 plane. As mentioned above, there is an array of filtered PIN diodes with peak energy responses from 1 to $40 \mathrm{keV}$. The low energy channels are sensitive to the line radiation from $\mathrm{H}$ - and He-like resonance lines and the high energy channels are sensitive to the thick target bremsstrahlung produced by hot electrons. The $x-$ ray signals from all channels peak at the same focus indicating that the peak line radiation occurs at maximum irradiance, since the hot electron production is an increasing function of the intensity ${ }^{4}$. Spectral measurements of the line radiation above $1 \mathrm{keV}$ are made with a flat crystal spectrograph, normally PET is used as the dispersive crystal. This device has an instrument width of $0.8 \mathrm{eV}$, determined by measuring the profile of the $K_{\alpha}$ line which has a known width. The time history of the emitted kilovolt radiation is measured with an $\bar{x}$-ray streak camera.

\section{DIAGNOSTICS OF THE LASER PULSES}

\subsection{Pulse length:autocorrellator:}

Since a net factor of two temporal broadening occurs from the 150-fsec dyeoscillator pulse to the 315-fsec amplified output pulse, the evolution of the pulse duration in the system is investigated using an auto-correlator. Autocorrelations of the pulse as it traverses the amplifier chain are presented in the Figure 3 These autocorrelations, obtained using twophoton ionization of DABCO vapor, all exhibit the 3:1 contrast ratio indicative of well-modelocked pulses. The $250 \mathrm{fsec}$ output pulse from the preamplifier (a) is broadened to $400 \mathrm{fsec}$ by the spatial filter. (b) An experimental investigation of this broadening reveals that it is caused not by the aperture in the spatial filter but by a combination of the spatial filter optics such as the dielectric mirrors ( $30 \mathrm{fsec}$ ), the $\mathrm{CaF}_{2}$ windows ( $60 \mathrm{fsec}$ ) and the fused silica lenses $(60 \mathrm{fsec})$. The effects of the final amplifier on the pulse width are twofold: With the amplifier turned off and evacuated, the pulse broadens from $400 \mathrm{fsec}$ to $540 \mathrm{fsec}$ (c) as a result of group velocity dispersion in the amplifier's four 0.95 $\mathrm{cm}$-thick quartz windows. This broadening is substantially larger than the 2-fsec broadening calculated for unchirped pulses. This result, along with the large time-bandwidth product $\Delta v \Delta \tau$ of 1.3 , indicates that the input pulse to the final amplifier is significantly chirped and can be subsequently compressed using passive components with negative net group-velocity dispersion. On the other hand, gain saturation during the amplification process compresses the pulse to a final width of $315 \mathrm{fsec}$ (d)

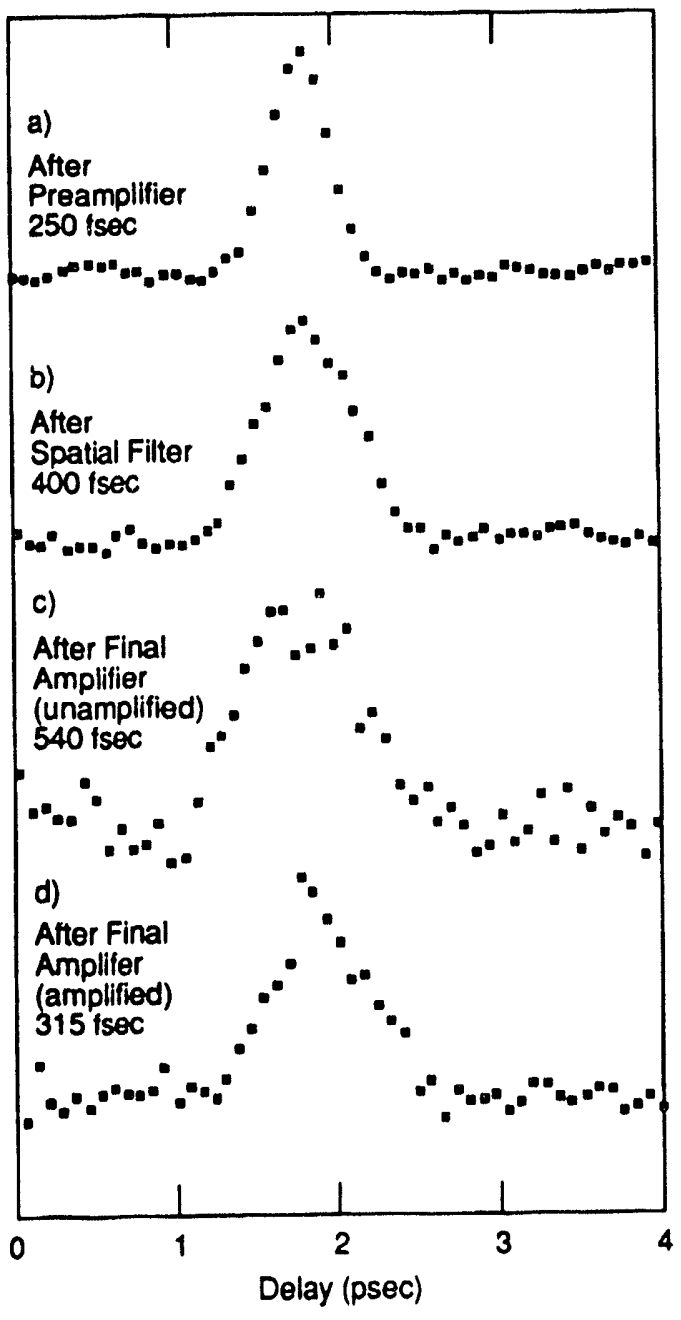

Fi.g. 3. Autocorelation traces at different points in the laser chain. 


\subsection{Pulse length:streak camera}

While an autocorellator is useful in measuring the details of short laser pulse in averaging mode, a streak camera has lower temporal resolution, but greater temporal coverage and the capability of recording the individual temporal history in a single shot. Thus a streak camera compements am autocorellator measurement. A UV streak camera with KI photo cathode has been used to measure the pulse length at the target. The streak camera entrance window has been designed to have the same thickness as the window of the target chamber, thus keeping any pulse-length stretching due to dispersion the same. The resolution of the streak camera and its dispersion have been measured on each shot by placing a known thickness of quartz in one half of the beam. Thus the pulse and its delayed counterpart are recorded simultaneously by the streak camera. One such record is shown in Figure $\mathbf{4}$ where multiple pulsing of the laser is shown.

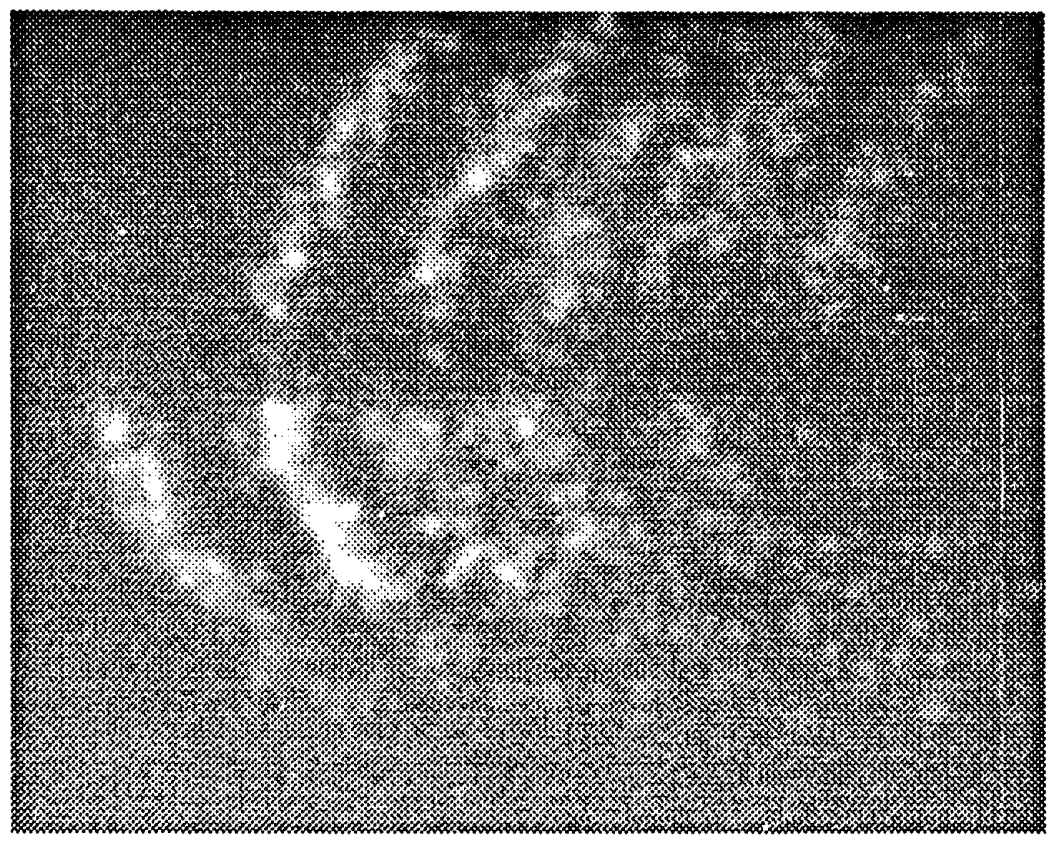

Fig. 4. A streak of the laser pulse. The distance between the pulses is roughly $19 \mathrm{ps}$.

The upper record was delayed by a $9.63 \mathrm{~mm}$ thick quartz. The shift between the two records correspond to the $18 \mathrm{psec}$ group delay in the quartz relative to the same thickness in air. The inter-pulse separation for this laser shot is $19 \pm 2 \mathrm{psec}$. While this record shows an extreme case for the operation of the laser, it shows one method of calibrating the variation of the streak camera dispersion across the full screen. For that shot the dispersion was $19 \mathrm{ps} / 53$ pixels at the left and $19 \mathrm{ps} / 63$ pixels at the right. The smallest recorded size was 4 pixels, the size of an MCP element in the intensifier screen. The streak camera was run at half its maximum streak rate.

\subsection{Wavelength content:}

Both the input and the back scattered spectrum of the laser pulse are measured on each shot. They are focused onto diffcrent spatial points on the input slit of a 0.5 meter spectrograph and they imaged onto separate areas of a 2-D OMA. This provides a simultaneous time integrated spectrum of both signals with a $0.25 \AA$ instrument width. The incident spectrum clearly shows the vibrational 
structure of the $\mathrm{XeCl}$ excimer system ${ }^{5,6}$ (see Figure 5). The back scattered spectra show significant blue shifts and are consistent with the existence of two separate phenomena. A low velocity shift of $10^{7} \mathrm{~cm} / \mathrm{sec}$ which is consistent with the bulk outward flow velocity deduced from x-ray pinhole photographs and a high velocity shift centered at $10^{8} \mathrm{~cm} / \mathrm{sec}$. Numerical simulations ${ }^{7}$ predict a peak outward velocity of the critical surface due to ionization of this same value. Therefore we postulate that the high velocity shift is due to an effective ionization wave that is generated in the low density region infront of the target. A similar behaviour has been observed in gaseous targets. ${ }^{8}$

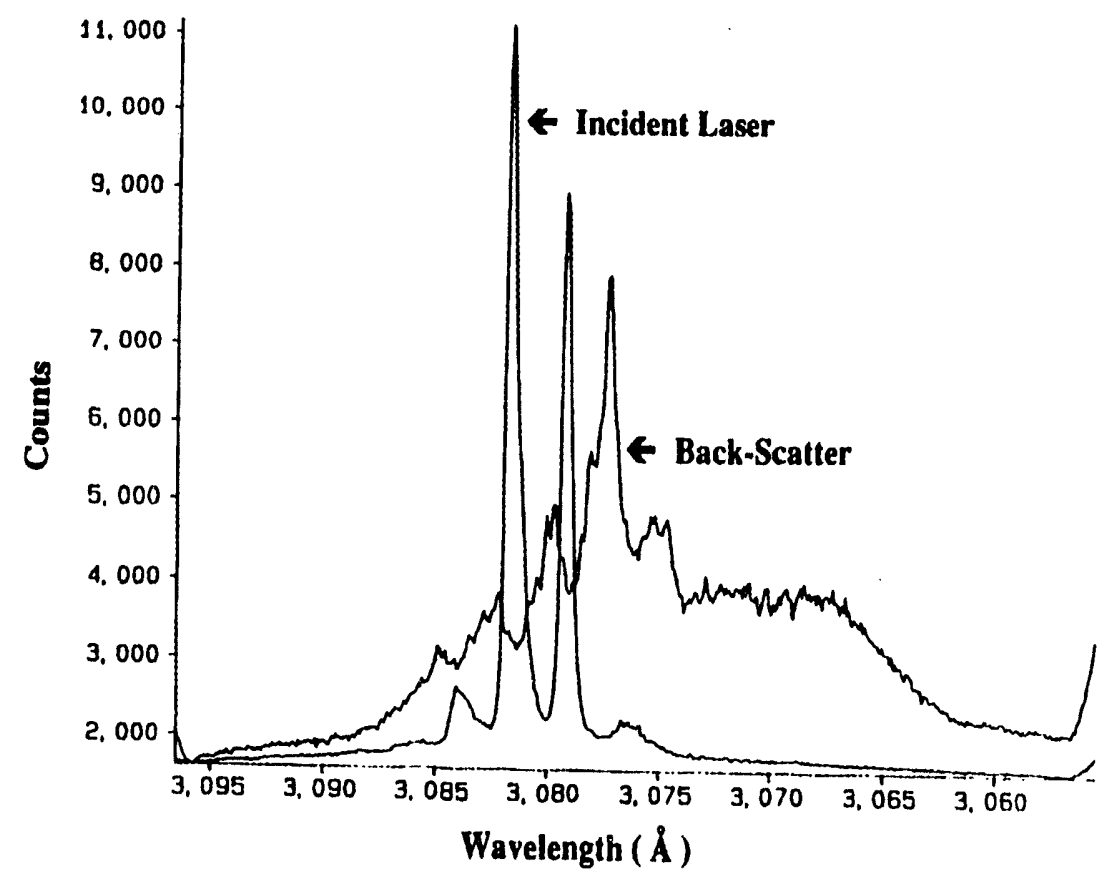

Fig. 5. Traces of the spectra of the incident laser light and that reflected from the plasma.

\subsection{Contrast of the prepulse:}

Contrast refers to the ratio of the peak laser power to the power that just preceeds the main pulse. The power that preceeds the main pulse can be of two sources; long term from the prelase or the ASE and short term due to multiple pulsing of the laser system. We refer in this section to the effect of the long prelase. The pulse contrast was measured using a pair of UV diodes. One diode observed the main pulse with an attenuation of $5 \mathrm{ND}$, while the other diode observed the ASE. The main pulse signal was integrated with a charge collector and calibrated versus an NBS traceable joule meter. The ASE diode was digitized with 10 samples/ns and the signal was calibrated using another NBS traceable joule meter. A typical prepulse trace is shown in Figure 6. The trace shows a prepulse before $22 \mathrm{~ns}$, when the main laser pulse arrives, and also shows the ASE lasting $20 \mathrm{~ns}$ after the main pulse. The main pulse contains $100 \mathrm{mj}$ and has a power of $3 \times 10^{11} \mathrm{~W}$. The prepulse contains $6 \mathrm{mj}$ and lasts $6 \mathrm{~ns}$. The peak prepulse power is $2 \times 10^{5} \mathrm{~W}$. The laser pulse power is at least six orders of magnitude larger than the prepulse power, and since its more focusable iots irradiance is much larger as well. The main laser-pulse power is off scale in Figure 6. 
Am plitude

$(m \vee)$

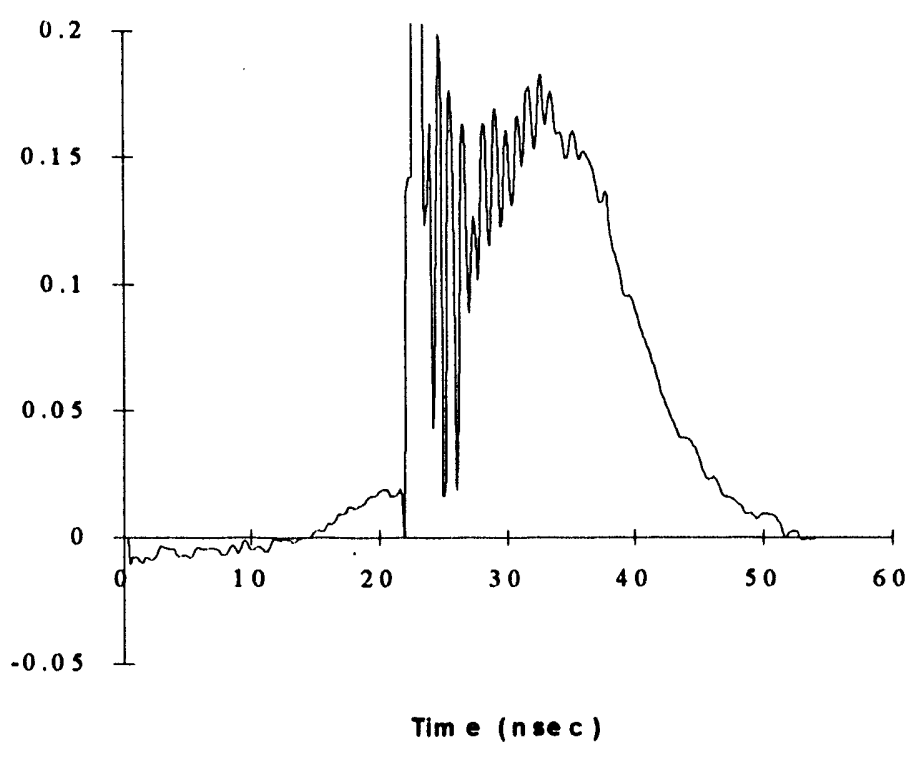

Fig. 6. A trace of the ASE from the laser. The jump occurs when the short $275 \mathrm{fsec}$ pulse is amplified. The bulk of the ASE arrives after the main laser pulse. The scale factor is $10.4 \mathrm{MW} / \mathrm{mV}$

\subsection{Quality of the laser beam:}

The beam quality of the fully amplified output pulse is determined by measuring its transmission through a calibrated pinhole when a heavily attenuated fraction is focused by the same focusing optics as used in target chamber. Alternatively, by imaging the incident laser beam in an equivalent focal plane with a $20 \mathrm{~m}$ focal length lens that minimizes the spherical aberrations at focus. Since the transverse profile of the unfocused beam is fairly flat-topped, we assume that the intensity profile in the focal plane is given by the Fraunhofer diffraction integral for a uniformly illuminated rectangular aperture. Using an on-axis parabolic mirror with an effective focal ratio of $\mathrm{f} / 3.7$, the measured transmission of $82 \%$ through a $10 \mu \mathrm{m}$ diameter pinhole then implies FWHM dimensions of the central diffraction spot of $3.4 \mu \mathrm{m} \times 4.1$ $\mu \mathrm{m}$. With these focal-spot dimensions our estimate for the mean irradiance is $4.9 \times 10^{18} \mathrm{~W} / \mathrm{cm}^{2}$. The peak irradiance, if the beam was diffraction limited, can be as high as $1 \times 10^{20} \mathrm{~W} / \mathrm{cm}^{2}$.

\section{DIAGNOSTIC OF THE LASER TARGET INTERACTIONS}

\subsection{Pinhole imaging}

An x-ray pinhole image of the silicon target emmitting region is shown in Figure 7. The region was observed through a 5 micron pinhole that is filtered by $\mathrm{xx}$ microns of beryllium, thus observing only the $\mathrm{H}$ - and He-like emission. The FWHM of the recorded image is less than $12 \mu \mathrm{m}$, which gives a deconvolved image of $7 \mu \mathrm{m}$. 


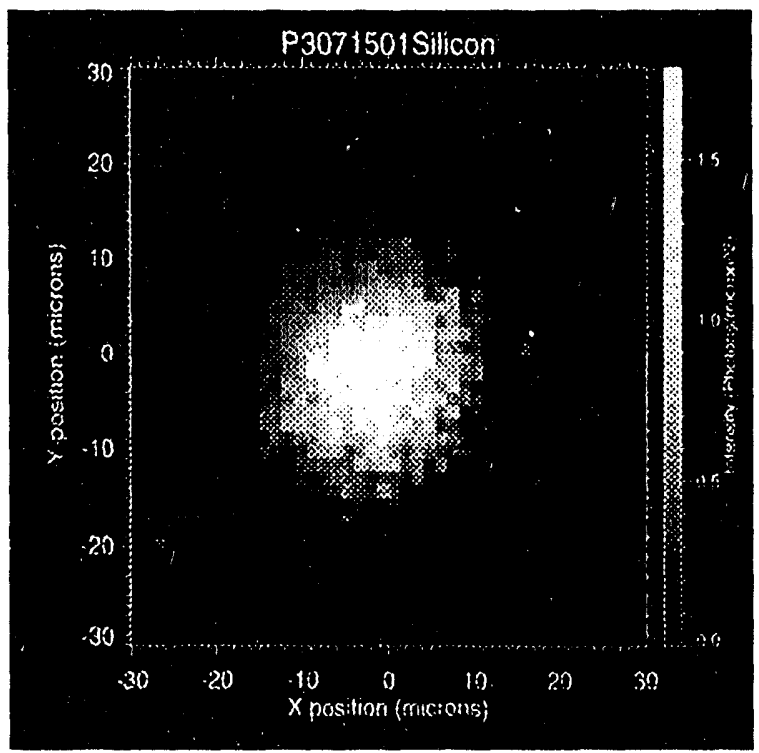

Fig. 7. A pihnole image of the $x$-ray emitting region.

\subsection{Visible imaging}

The purpose of the visible imaging is to investigate the stability of Gbar shock waves in a solid, and the formation of holes through a foil target. Previous work ${ }^{9}$ in gases has shown that Taylor-Sedov blast waves propagating through nitrogen are stable, but an instability develops for propagation in xenon. This raises the question whether a similar material dependence occurs for shock wave propagation in solids. The experimental setup and associated diagnostics are shown in Fig.7. A thin silicon foil target $(5-10 \mu \mathrm{m})$ is placed at the focus of a F/1.7 parabola and is irradiated by a 300 fs laser pulse at severai $10^{18} \mathrm{~W} / \mathrm{cm}^{2}$. The radiation pressure and thermal pressure after absorption generate a Gbar shock which propagates into the foil.

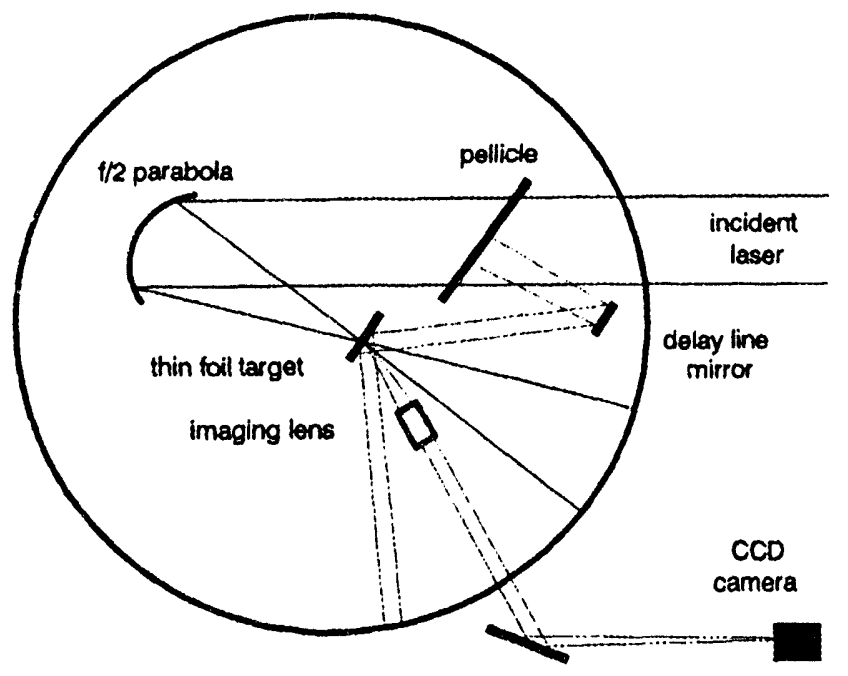

Fig. 7. Schematic diagram of the imaging layout 
A pellicle in the incident beam in front of the parabola splits off a small fraction of the laser pulse which is then steered to illuminate the back of the foil. By adjusting the distances from the pellicle between the front and back of the foil, the time between the initiation of the shock on the front and the snap-shot illumination on the back can be varied. Hence the shock breakout conditions on the back surface can be imaged with a single 300 fsec snap-shot at a specific time during the breakout.

The imaging system consists of an F/2.7 lens, color corrected over $2 \mathrm{~nm}$ at the laser wavelength of $308 \mathrm{~nm}$ at a magnification of 30 . The resolution of the lens in the object plane is about $1 \mu \mathrm{m}$, which corresponds to 3 pixels in the $10-\mu \mathrm{m}$ pixel CCD camera. The images are recorded for analysis by an 8-bit digital recording system. Figure 8 shows a typical image taken about $200 \mathrm{psec}$ after initiating the shock on the front of the foil and for comparison, the image of the final hole. It appears that in this shot we captured the image during the formation of the hole.

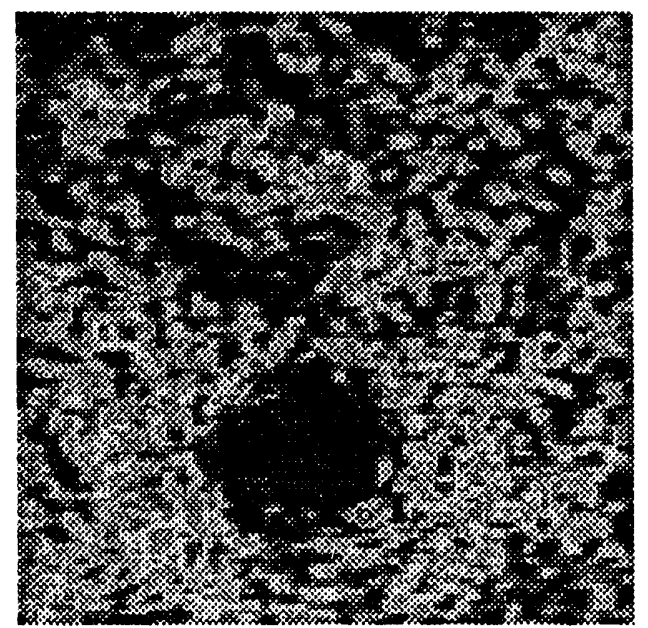

Fig. 8a. Breakout imaged with a 315 fsec pulse $200 \mathrm{psec}$ after the main pulse.

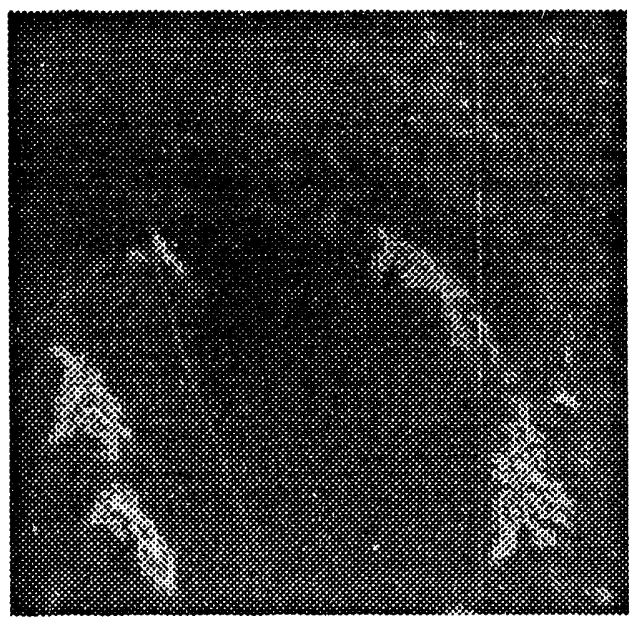

Fig. 8b. Image of the final hole. The size of the image is $160 \mu \mathrm{m}$ on a side.

\subsection{Soft-x-ray production}

The soft $\mathrm{x}$-rays in the wavelength range 20-60 Angstroms are recorded with a flat-field imaging spectrometer ${ }^{10,11}$. The spectrometer uses two variable line spacing gratings to record the spectrum; the first has a resolution of 8000 and the second has a resolution of 200. The spectrum is detected and amplified by a $7 \mu \mathrm{m}$ diameter MCP that is coated with CsI. The output of the MCP is coupled by a fiber optic taper to a one-dimensional silicon diode array. The spectrometer is absolutely calibrated at a few wavelengths using an electron impact $x$-ray source. A typical raw spectrum of a silicon target, when the laser is focused in front of the target, is shown Figure 9 


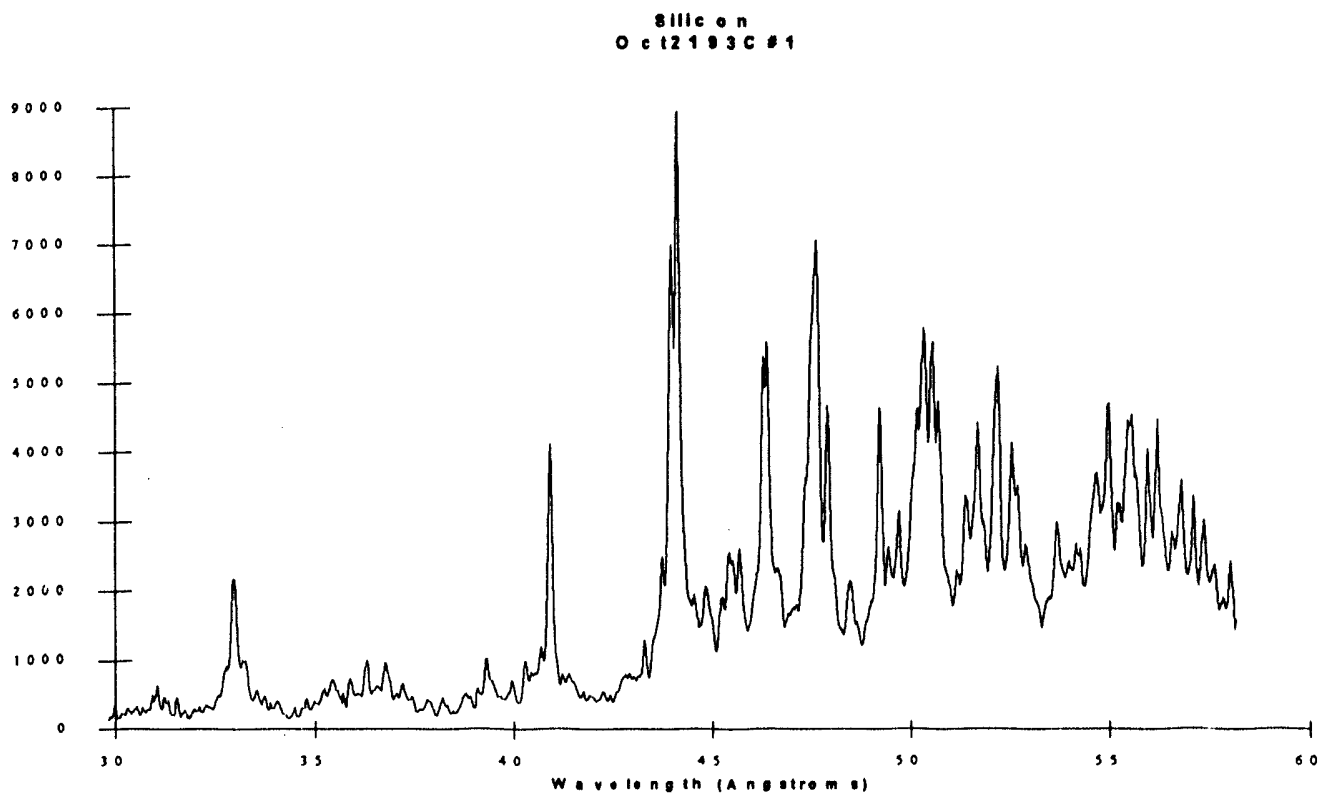

Fig. 9. X-ray spectrum of the $\mathrm{Li}$ - and Be- like lines from silicon

For that shot we measure mostly the Lithium like lines from the silicon target. Most of the lines are $1 s^{2} 31-1 s^{2} 2 p$. The He- and H-like line are weak in that shot. The slope of the continuum around 58 Angstroms can be used to estimate a time-average temperature of the plasma, $30 \mathrm{eV}$. When the laser is focused better, the spectrum shows the Balmer series of the silicon target as well. The timc-average temperature of this shot is $180 \mathrm{eV}$

\section{Silic on}

\section{Aug 293A\#2}

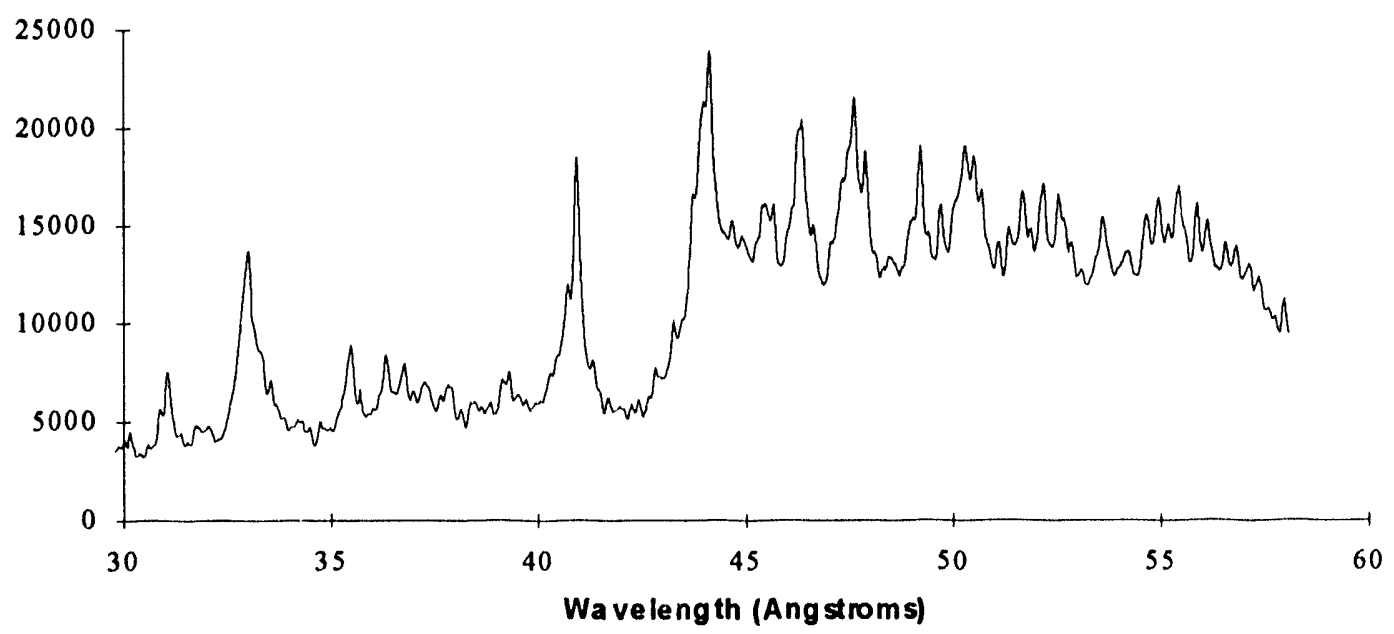

Fig. 10. X-ray spectrum of the He- and Li- like lines from Silicon 


\subsection{Imaging $X$-ray spectrometry}

The Lyman series of lines from $\mathrm{H}$-like and He-like silicon are observed using a spherically bent crystal spectrometer. The spectrometer, built by Faneov et al., ${ }^{12}$ has a resolution $>8000$ at the $\mathrm{H}-$ like $\mathrm{L}_{\alpha}$ line. Figure 11 show a silicon spectrum measured with two laser shots. The spectrum shows the satellites to the $\mathrm{L}_{\alpha}$ line, which were identified as He-like dielectronic satellites $2121^{\prime}-1 \mathrm{~s} 21$, and $2131^{\prime}-1 \mathrm{~s} 31$. The The relative intensities of the lines can be used to derive an electron temperature of the plasma of 460 $\mathrm{eV}$.

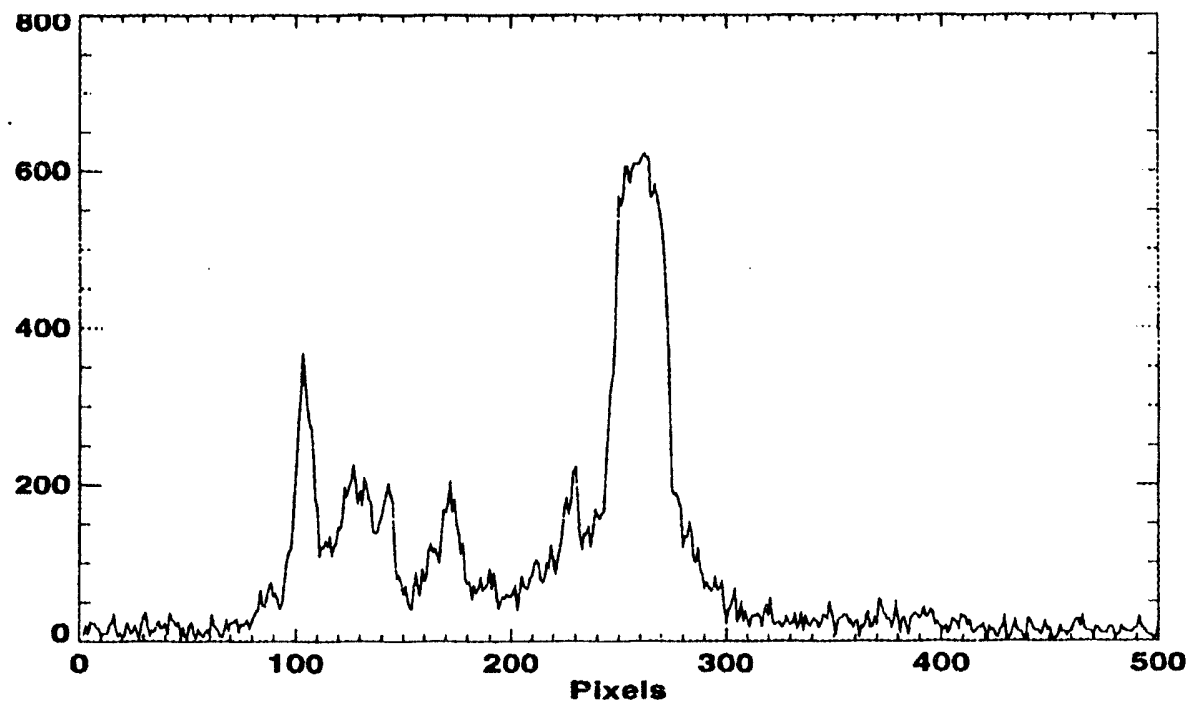

Fig. 11. Curved Crystal Specrometer spcctrum of the H-like Lyman alpha line.

\subsection{X-ray Pulse Length}

The $\mathrm{x}$-rays that are generated in the plasma are observed with an $\mathrm{x}$-ray streak camera with $\mathrm{KI}$ pholo cathode. The streak camera observes the plasma through several foils ${ }^{13}$ each with observes a particular energy region. Two typical spectra, through 4 foils that cover different spatial extents of the slit are shown in Figure 12a and 13a. The corresponding laser pulse histories are shown in Figure $12 \mathrm{~b}$ and $13 \mathrm{~b}$ respectively. Figure 12 shows that a single $\mathrm{x}$-ray pulse is generated when the laser has one pulse. When the laser has two pulses, Figure 13, but with the same energy as the single pulse, then the generated $\mathrm{x}$-rays show an equal number of pulses, separated by the same $19 \mathrm{psec}$ time. However note that while the second UV pulse is weaker than the first, the second $x$-ray pulse is not much weaker than the first $x$-ray pulse. The first pulse may generate few hundred $\mathrm{eV}$ plasmas with expansion velocities of $3 \times 10^{7} \mathrm{~cm} / \mathrm{sec}$. By the time the second pulse arrives the plasma expands another $6 \mu \mathrm{m}$ and the second pulse interacts with even lower density electrons and atoms. X-ray calorimetry, using filtered silicon diodes, indicates that the multiple pulse shot generated $50 \%$ more line radiation than the single pulse shot. The hard $\mathrm{x}$-rays show a $20 \%$ increase. The calorimetry results agree with the results from the $x$-ray streak camera measurements. 


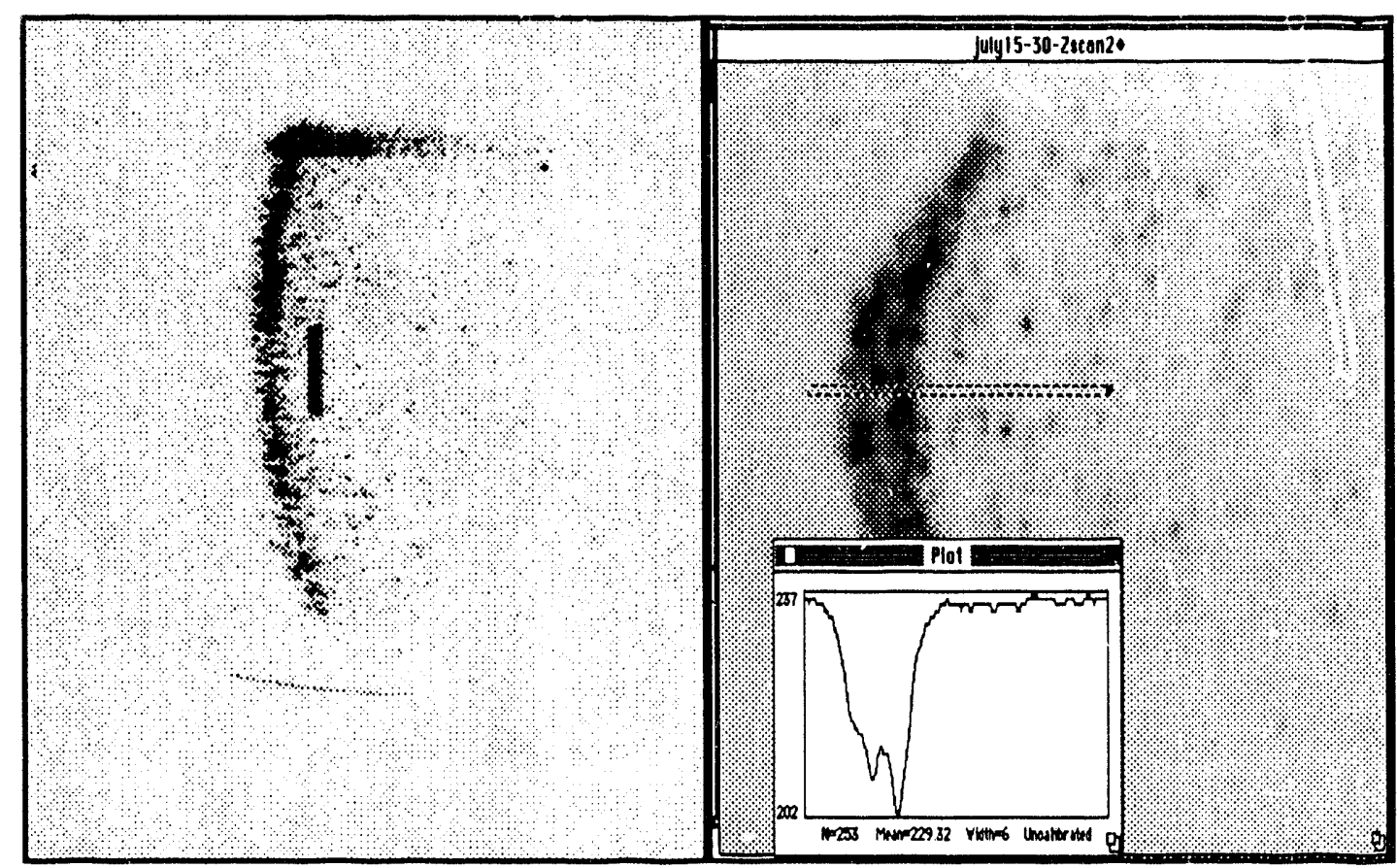

Fig. 12a. X-ray streak camera record when the laser generates a single pulse.

Fig. 12b. UV streak camera record when the laser generates a single pulse.

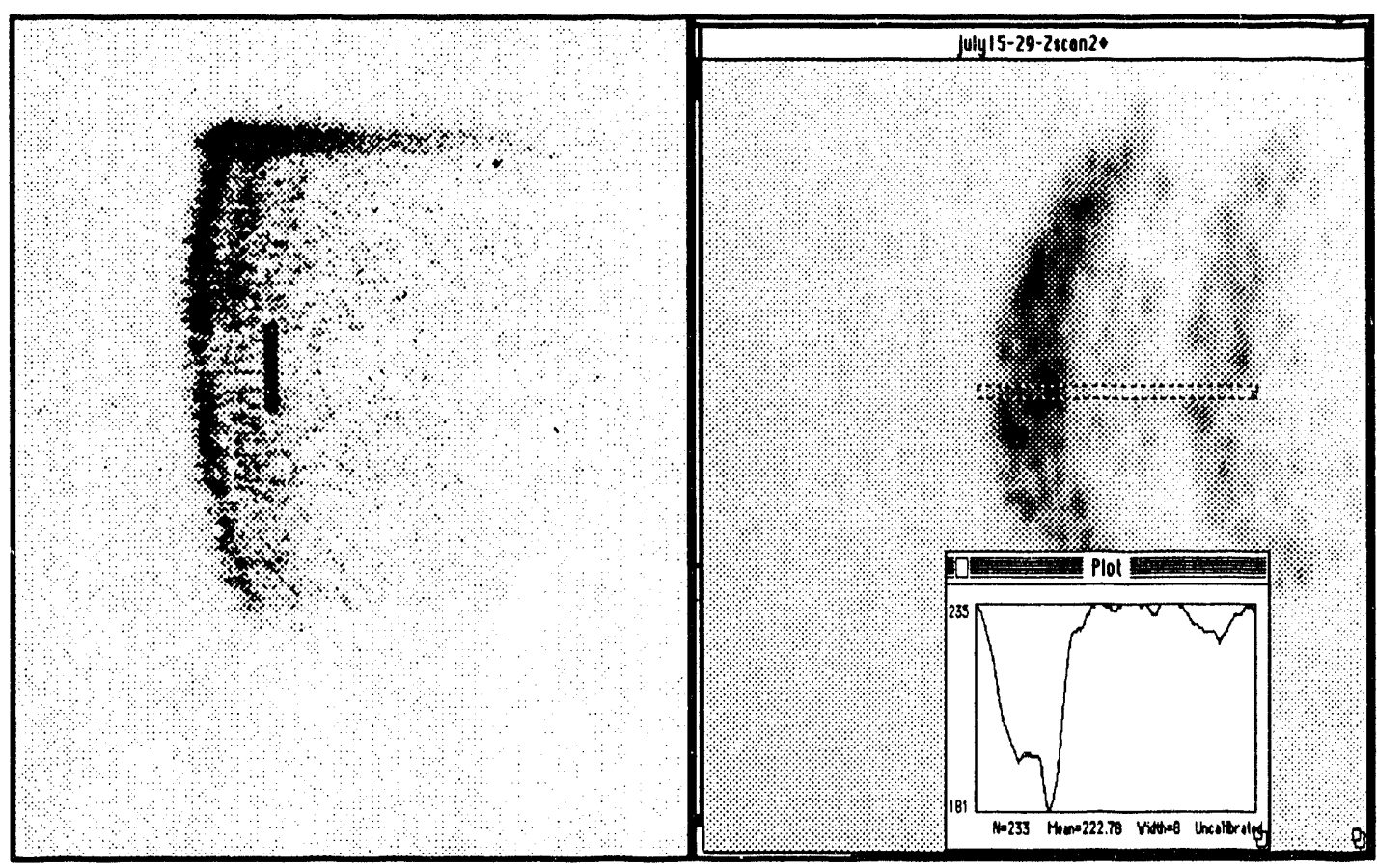

Fig. 13a. X-ray streak camera record when the laser generates a two pulses.

Fig. 13b. UV streak camera record when the laser generates two single pulses. 


\section{SUMMARY}

We have shown that a variety of diagnostics are needed and used to characterize the various laser properties: prepulse, pulse length, energy and spot size. Similarly many properties of X-rays from the laser generated plasmas are recorded simultaneously: pulse length, energy content, spatial extent and spectral content. All these are needed in order to understand how the laser energy is coupled to a target in order to generate $x$-rays. We found that a small amount of ASE is needed to increase the $x$-ray production. We also found that the existence of a large first pulse, as opposed to the longer prelase or ASE, does not significantly reduce the production of $x$-rays from the second pulse, at least if the interpulse separation is greater than the laser pulse width. Indeed, the line-conversion efficiency from the second pulse is enhanced over that without a prepulse.

\section{ACKNOWLEDGMENTS}

We would like to ackiowledge the expert contributions of Jan Studebaker and Richard Eitland in running the diagnostics, of Keith Hosak in running the laser, of Bernie Carpenter in film handling, Craig Mishenko in visible imaging, and of David Orro in some of the image handing.

\section{REFERENCES}

I. A. J. Taylor, C. R. Tallman, J. P. Roberts, C. S. Lester, T. R. Gosnell, P. H. Y. Lee, and G. A. Kyrala, "High-intensity subpicosecond XeCl laser system," Opt. Lett. 15, 39-41 (1990).

2. J.A. Cobble, G. T. Schappert, L. A. Jones, A. J. Taylor, G. A. Kyrala, and R. D. Fulton, "The interaction of a high irradiance, subpicosecond laser pulse pulse with aluminum: The effect of the prepulse on x-ray production," J. Appl. Phys. 69, 3369-3371 (1991).

3. D.E. Casperson, J. A. Cobble, R. D. Fulton, G. A. Kyrala, G. T. Schappert, A. J. Taylor and E. Wahlin, "Thin foil x-ray converters pumped by subpicosecond excimer lasers," J. Appl. Phys. 74, 3707-3711 (1993).

${ }^{4}$. W. Priedhorsky, et al., Phys. Rev. Lett. 47, 1661(1981).

5. B. Dick, et al., Optics Comm. 62, 277 (1987).

6. A.J. Taylor, et al., in Ultrafast Phenomena VII, (Springer-Verlag, Berlin, 1991), p. 104.

7. G. Olsen et al., private communications

8. DOWNER ET AL.

9. J. Grun et. al. Phys. Rev. Lett. 66, 2738, (1991)

${ }^{10}$. M. Hettrick, "Optical Systems for high resolution spectroscopy," U. S. Patent No. 4,776,696 (1988).

11. M. Hettrick, "High resolution grating for soft x-rays," Nuclear Instruments and Methods A266, 404413 (1988).

12. B. A. Bryunetkin, S.A. Pikuz, I. Y. Skobelev and A. Y. Faenov, "Imaging spectroscopy of hightemperature plasma sources," Laser and Particle Beans, 10, 849-860 (1992)

13. J. A. Cobble, R. D. Fulton, L. A. Jones, G. A. Kyrala, G. T. Schappert, A. J. Taylor and E. K. Wahlin, "X-ray streak camera diagnostic of picosecond laser-plasma interactions," Review of Scientific

Instruments, 63, 5116-5118 (1992). 
